\title{
Cyclophanes
}

\section{Selective Synthesis of Hetero-Sequenced Aza-Cyclophanes}

\author{
Matthias Otte, ${ }^{*[a, b]}$ Martin Lutz, ${ }^{[c]}$ and Robertus J. M. Klein Gebbink ${ }^{[a]}$
}

\begin{abstract}
The selective synthesis of purely organic cages with hetero-sequenced functionalized cavities is demonstrated. The strategy to obtain these compounds is based on a stepwise approach using thermodynamically controlled imine condensations. To accomplish this, the amine building blocks carry additional azide groups, which act as masked amines and enable the synthesis of the desired cavities. This approach offers a pre-
\end{abstract}

cise control of interior functionalization, the substitution pattern and the cage size, which is demonstrated by the selective synthesis of four cages. The largest described cage has been investigated towards its ability for guest encapsulation and is able to selectively encapsulate functionalized arenes that offer a matching substitution pattern.

\section{Introduction}

The development of functional molecular cage compounds with defined cavities and pores is of major importance in modern chemistry. To date, such cages have found numerous applications including their use as hosts for selected compounds, ${ }^{[1]}$ for gas storage, ${ }^{[2]}$ as molecular switches, ${ }^{[3]}$ to stabilize highly reactive reagents ${ }^{[4]}$ or as catalysts. ${ }^{[5]}$ Synthetic approaches towards these compounds involve very often self-assembly through metal-ligand coordination ${ }^{[6]}$ or hydrogen-bonding interactions. ${ }^{[7]}$ Due to self-assembly phenomena, the desired cage compounds are often obtained in high yields. They are usually highly symmetric, resulting in a highly symmetric interior functionalization. Notably, interior functionalization is of great importance as it is together with exterior functionalization and pore design the major handle to steer the cage function.

The selective synthesis of less symmetrical cavities is thus a great challenge for the chemistry in cavities. One approach of great interest is to obtain less symmetrical cages through metal-ligand coordination. Examples of those coordination cages have been recently obtained by so-called social self-sorting. ${ }^{[8]}$ However, the occurrence of social self-sorting is currently hard to predict as well as the structure of the thereby formed product. In addition, social self-sorting often comes with the cost of a different cavity geometry compared to the symmetrical cages, as building blocks of different shapes need to be employed. Furthermore, unsymmetrical cages can sometimes

[a] Organic Chemistry \& Catalysis, Debye Institute for Nanomaterials Science, Faculty of Science, Universiteit Utrecht,

Universiteitsweg 99, 3584 CG, Utrecht, The Netherlands

[b] Institut für Anorganische Chemie, University of Goettingen,

Tammanstrasse 4, 37077 Göttingen, Germany

E-mail: matthias.otte@chemie.uni-goettingen.de www.matthiasottelab.com

[c] Crystal and Structural Chemistry, Bijvoet Center for Biomolecular Research, Faculty of Science, Utrecht University,

Padualaan 8, $3584 \mathrm{CH}$ Utrecht, The Netherlands

$\square$ Supporting information for this article is available on the WWW under http://dx.doi.org/10.1002/ejoc.201700106. only be obtained by substitution of building blocks from an already assembled highly symmetric cage.

Purely organic covalent cages might be an alternative to metal-coordination cages to create cavities of lower symmetry. ${ }^{[9]}$ However, examples for the selective synthesis of cages that offer a less symmetrical interior are rare and use reactions that occur under kinetic control. ${ }^{[10,11]}$ Those reactions bear the disadvantage that once a bond that leads to an undesired oligomer has been formed it cannot be repaired. The appearance of dynamic combinatorial chemistry (DCC) made the synthesis of numerous new purely organic macrocycles and cages possible as this concept allows the high yielding synthesis of the desired organic compounds under thermodynamic control. ${ }^{[12]}$ DCC is an important addition to the portfolio of cavity design, which has so far been dominated by approaches based on supramolecular interactions. Zhang and coworkers reported that DCC offers the opportunity to synthesize hetero-sequenced macrocycles with unsymmetrical cavities through imine condensation followed by olefin metathesis. ${ }^{[13]}$ Martinez and Dufaud reported the synthesis of catalytically active $1+1$ cages by using a reductive amination strategy. ${ }^{[14]}$

$3+2$ aza-cyclophanes are important organic cage compounds, which have demonstrated great ability for the selective encapsulation of sugars. ${ }^{[15]}$ In addition, the porosity of the analogous $6+4$ aza-cyclophanes has been widely investigated. ${ }^{[16]}$ Moreover, $\beta$-diketiminate-(nacnac)-functionalized aza-cyclophanes form first-row transition-metal complexes that can activate small molecules such as $\mathrm{N}_{2}$ and $\mathrm{CO}_{2} \cdot{ }^{[17,18]}$ While the synthesis of numerous symmetrical $3+2$ aza-cyclophanes have been reported by now, ${ }^{[19]}$ the selective synthesis of the analogous and less symmetric $2+2+1$ or $2+1+2$ cages has not been described as statistical mixtures are obtained. Reported approaches to less symmetrical aza-cyclophanes so far employ the installation of amide instead of amine groups or use a combination of amide and amine groups. ${ }^{[9,10]}$ To accomplish those cage synthesis, low yielding kinetically controlled reactions leading to macrocyclic precursors or the final cage products are employed. 


\section{Results and Discussion}

Based on the importance of aza-cyclophanes and our interest in the design of precisely functionalized hetero-sequenced cavities, $^{[20]}$ we were wondering if the selective synthesis of the $2+2+1$ aza-cyclophane 1 can be achieved by using a modified reductive amination protocol (Scheme 1a). To accomplish the synthesis of $\mathbf{1}$ we envisioned a three-step synthetic approach from known building block 2, which has two benzylamine groups and one benzyl azide functionality. ${ }^{[21]} \mathbf{2}$ can be synthesized in three steps starting from commercially available 1,3,5triethylbenzene. We chose $\mathbf{2}$ because its azide functional group is known to tolerate the reaction conditions for the reductive amination. ${ }^{[21]}$ As azides can be transformed into amines, one could see the azide in $\mathbf{2}$ as a masked amine. When reacting a 1:1 mixture of $\mathbf{2}$ and 2,6-pyridinecarboxyaldehyde (3) for $20 \mathrm{~h}$ at room temperature, followed by the addition of $\mathrm{NaBH}_{4}$, the desired macrocyclic product 4 was obtained with $55 \%$ yield. With 4 in hand, its transformation into the corresponding amine was investigated. As the substrate contains benzylamine functionalities we avoided the use of a palladium hydrogenation catalyst and applied a Staudinger reaction protocol instead. Addition of four equivalents triphenylphosphine to $\mathbf{4}$ in THF, followed by the addition of water and purification with column chromatography gave 5 in a yield of $74 \%$.

With 5 in hand, its ability to form a 2+2+1 aza-cyclophane was investigated. Gratefully, addition of one equivalent of 4tert-butyl-2,6-diformylphenol (6) to one equivalent of $\mathbf{5}$ gave the hetero-sequenced $2+2+1$ cage 1 in $91 \%$ yield after in situ reduction of the formed imines. $\mathbf{5}$ is likely to adopt a conformation, which favours cage closing reaction with $\mathbf{6}$ over the com- a)

\section{Control: Functionalization}

2

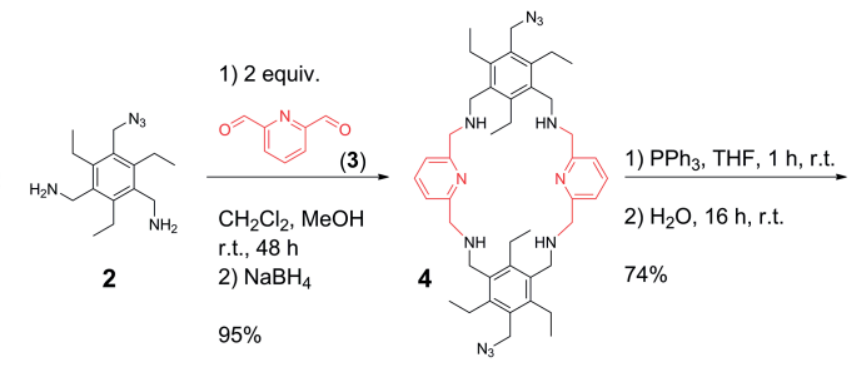

Control: Substitution pattern

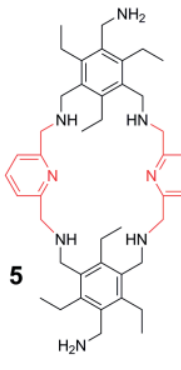

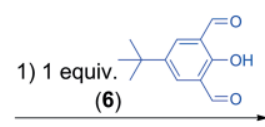

$\mathrm{CH}_{2} \mathrm{Cl}_{2}, \mathrm{MeOH}$, r.t., $20 \mathrm{~h}$ 2) $\mathrm{NaBH}_{4}$

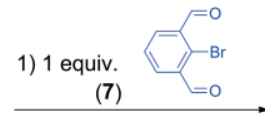

$\mathrm{CH}_{2} \mathrm{Cl}_{2}, \mathrm{MeOH}$, r.t., $20 \mathrm{~h}$ 2) $\mathrm{NaBH}_{4}$
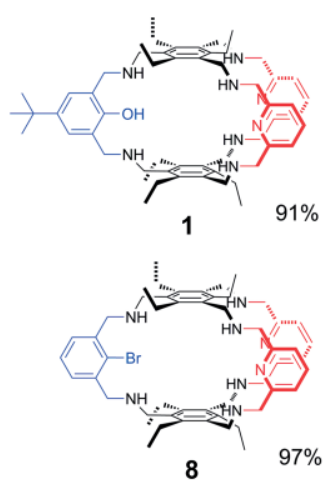

b)
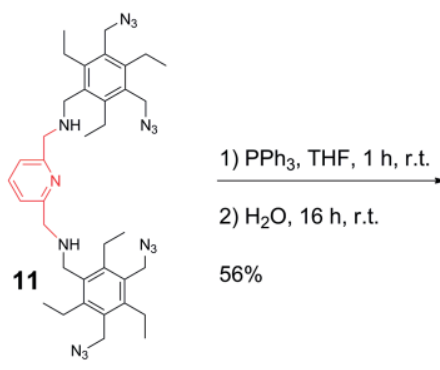
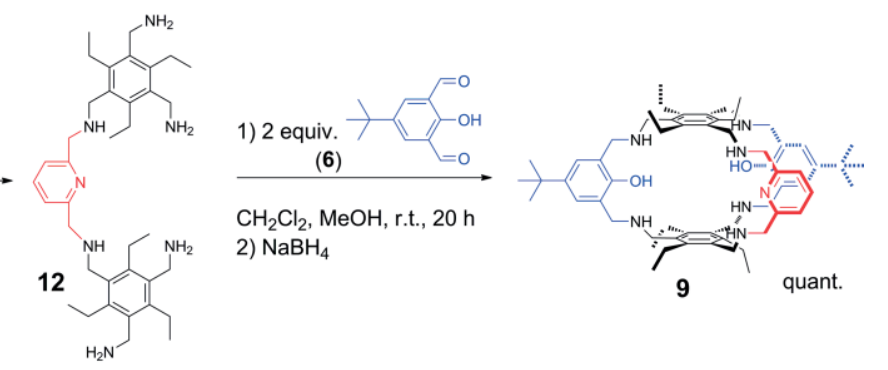

\section{Control: Cage size}

c)

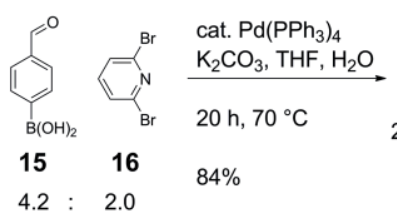

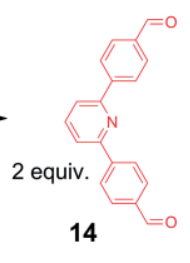
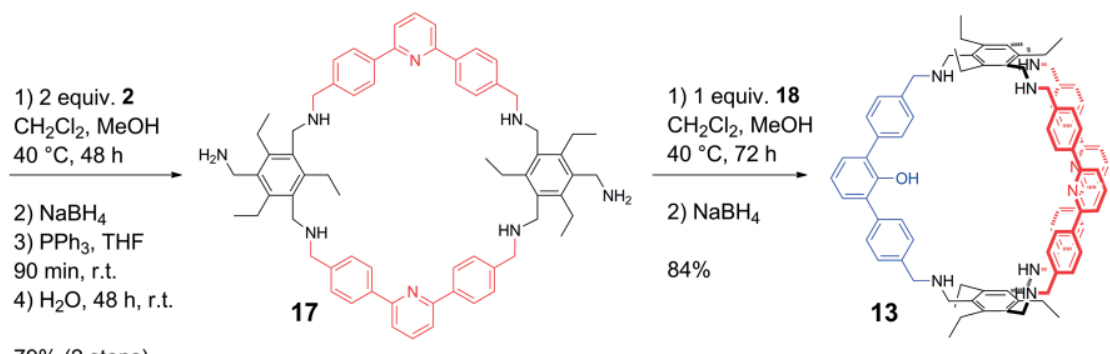

$79 \%(2$ steps $)$
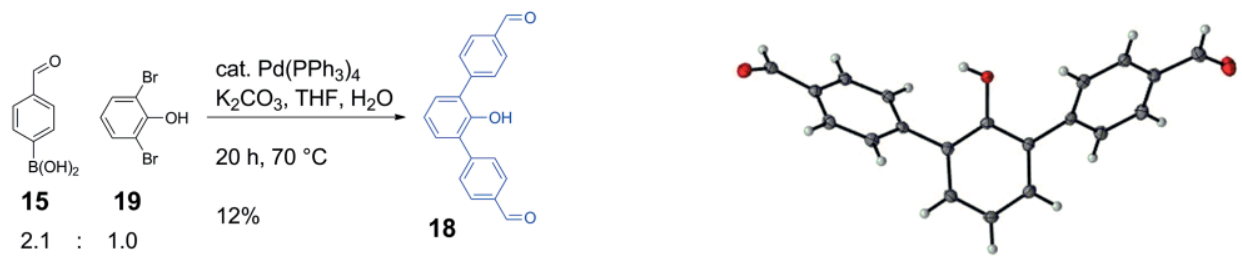

Scheme 1. Synthesis of hetero-sequenced cages 1, 8, 9 and $\mathbf{1 3}$ and molecular structure of $\mathbf{1 8}$ in the crystal (see the Supporting Information, Figure S1). 
peting oligomerization. Cage $\mathbf{1}$ was characterized by using mass spectrometry and NMR and IR spectroscopy. ESI-MS analysis revealed the formation of 1 by showing signals with $\mathrm{m} / \mathrm{z}$ values of 879.6398 and 440.3180 that correspond to $1+\mathrm{H}^{+}$and $\mathbf{1}+2 \mathrm{H}^{+}$. Furthermore, host-guest complexes like $\mathbf{1}+2 \mathrm{H}_{2} \mathrm{O}+\mathrm{H}^{+}$ have been observed during ESI-MS analysis. It is very important to note that no symmetrical $3+2$ cages, such as the all phenol or the all pyridine cage, nor the corresponding $2+1+2$ ( 1 pyridine; 2 phenol) cage have been observed. Interestingly, a minor signal at $\mathrm{m} / \mathrm{z}$ at 1759.2037 was observed that corresponds to $2^{*} \mathbf{1}+\mathrm{H}^{+}$. To see if this stems from the formation of a larger $4+4+2$ cage or if it might be a cluster in the gas phase, DOSY NMR spectroscopy was performed. The DOSY NMR spectrum indicated only formation of products of one size, which rules out the formation of significant amounts of the $4+4+2$ cage. Further NMR analysis gave additional support for the selective formation of $\mathbf{1}$ (see the Supporting Information). To prove the generality of this approach, we substituted 6 for 2-bromoisophthalaldehyde (7) (Scheme 1a). We were delighted to observe the formation of the bromo-functionalized cage $\mathbf{8}$ with an excellent yield of $97 \%$. In this regard, our modular approach enables the precise control of cage functionalization, which is accomplished by the introduction of functional groups at a late synthetic stage.

As we demonstrated the selective synthesis of the $2+2+1$ cages $\mathbf{1}$ and $\mathbf{8}$, we became interested if the selective synthesis of the $2+1+2$ cage 9 could be accomplished in a similar way. We started our synthetic approach with building block 10 (Scheme 1b), which was obtained as a side product from the synthesis of $\mathbf{2}^{\left[{ }^{[21]}\right.}$ We reacted two equivalents of $\mathbf{1 0}$ with one equivalent of $\mathbf{3}$, followed by reduction with $\mathrm{NaBH}_{4}$. After purification by column chromatography, $\mathbf{1 1}$ was obtained with quantitative isolated yield. The following Staudinger reaction gave the tetraamine 12 after column chromatography in $56 \%$ yield. The cage synthesis was finalized through a reductive amination of one equivalent of $\mathbf{1 2}$ with two equivalents of $\mathbf{6}$. The desired cage 9 was obtained in a quantitative yield and was characterized by ESI-MS, ${ }^{1} \mathrm{H}$ NMR, ${ }^{13} \mathrm{C}$ NMR and IR spectroscopy (see the Supporting Information). Similar to $\mathbf{1}$, signals corresponding to $\mathbf{9}+\mathrm{H}^{+}$and $\mathbf{9}+2 \mathrm{H}^{+}$at $\mathrm{m} / \mathrm{z}$ values of 950.7000 and 475.8539 were observed. No formation of undesired cage 1 or any $3+2$ cages was observed. It should be noted that a small signal corresponding to $2^{*} \mathbf{9}+\mathrm{H}^{+}$or the $4+2+4$ cage was again observed

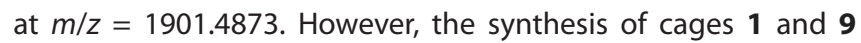
demonstrates that our approach is suitable to control the functionalization pattern in hetero-sequenced purely organic cages.

As we have shown that the selective synthesis of 1, 8 and $\mathbf{9}$ is possible, we were wondering if our approach might also be suitable to obtain larger purely organic and hetero-sequenced cages. To test this hypothesis we envisioned synthesizing the 2+2+1 cage 13 (Scheme 1c). Cage 13 has additional phenyl groups between the amine linker and the pyridine/phenol moieties, which results in a larger cavity. We started the synthesis of the pyridine-containing building block 14 with a Suzuki coupling reaction of 4-formylphenylboronic acid (15) and 2,6-dibromopyridine (16). Applying a reductive amination with $\mathbf{2}$, followed by a Staudinger reaction, $\mathbf{1 4}$ was transformed into mac- rocycle 17 . To complete the cage synthesis, building block 18 was synthesized from 2,6-dibromophenol (19) and 15 in $12 \%$ yield by using a Suzuki coupling reaction. Compound $\mathbf{1 8}$ was obtained as a yellow/greenish powder, which is fluorescent in $\mathrm{CDCl}_{3}$. When an NMR sample of $\mathbf{1 8}$ was kept standing for several days, crystals suitable for X-ray diffraction were obtained (see the Supporting Information for a detailed discussion of the crystal structure). With $\mathbf{1 7}$ and $\mathbf{1 8}$ in hand the reductive amination towards cage $\mathbf{1 3}$ was investigated. Performing the reaction at $40{ }^{\circ} \mathrm{C}$ resulted in the formation of a major and a minor fraction, which could be separated by using column chromatography. Mass spectrometry investigations of the major fraction revealed a signal at the $\mathrm{m} / \mathrm{z}$ value of 1279.8217 that corresponds to $13+\mathrm{H}^{+}$. In addition, the $\mathrm{m} / \mathrm{z}$ values of $640.4017,427.2672$ and 320.7004 that correspond to the higher charged species $13+2 \mathrm{H}^{+}, 13+3 \mathrm{H}^{+}$and $13+4 \mathrm{H}^{+}$were observed as well. Furthermore, DOSY NMR spectroscopy confirmed that $\mathbf{1 3}$ is a single compound (see the Supporting Information). The formation of 13 was further confirmed by ${ }^{1} \mathrm{H}$ NMR, ${ }^{13} \mathrm{C}$ NMR and IR spectroscopy (see the Supporting Information). Given our analysis we conclude that $\mathbf{1 3}$ has been isolated within $84 \%$ yield. In addition to 13 , we were also able to separate a second fraction. ${ }^{1} \mathrm{H}$ NMR signals of this fraction are unfortunately very broad. Mass spectrometry analysis revealed signals of a double charged species with an $\mathrm{m} / \mathrm{z}$ value of 1279.9606 that corresponds to $2 * 13+2 \mathrm{H}^{+}$. The yield of $2 * 13$ would be $15 \%$. The structure of this compound is currently under further investigation. Although $2^{*} 13$ has been formed as a side product, the synthesis of 13 with an isolated yield of $84 \%$ demonstrates that our approach is also suitable to control the cage size.

With 13 in hand we investigated if the hetero-sequenced functionalized cavity might be suitable to host functionalized guests. As similar cages are suitable hosts for sugars, ${ }^{[10,15]}$ we started our investigations with the study of the encapsulation behaviour of several poly-functionalized natural products (Table 1). Unfortunately, neither $\beta$-lactose, nor the amino acids L-tyrosine and L-lysine (entries 1-3) gave any indication to be encapsulated within 13. We then turned our focus towards the encapsulation of functionalized arenes because we assumed that the planar nature of those might favour the interaction with the functional groups of the cage interior. We envisioned that compounds with proton donor and acceptor moieties

Table 1. Study of $\mathbf{1 3}$ as host for functionalized guests.

\begin{tabular}{lll}
\hline Entry $^{[a]}$ & Guest & Encapsulation observed \\
\hline 1 & 3-lactose & no \\
2 & L-tyrosine & no \\
3 & L-lysine & no \\
$\mathbf{4}$ & 3-hydroxypyridine (20) & yes \\
5 & 2,6-dipyridinecaboxylic acid & no \\
6 & pyridine & no \\
7 & phenol & no \\
8 & 3-iodopyridine & no \\
9 & 2-hydroxypyridine & no \\
$\mathbf{1 0}$ & resorcinol (21) & yes \\
11 & catechol & no
\end{tabular}

[a] All experiments at room temperature in $\mathrm{CDCl}_{3}$; for detailed information see the Supporting Information. 
might be well suited as guests because 13 offers a complementary cavity functionalization.

Indeed, when 13 and 3-hydroxypyridine (20) were mixed in a 1:1 ratio in $\mathrm{CDCl}_{3}$, significant downfield shifts of signals corresponding to $\mathbf{2 0}$ were observed, which indicate the formation of 13@20 (Figure 1a-c; Table 1, entry 4). Signal a shifted from $8.32 \mathrm{ppm}$ in free $\mathbf{2 0}$ to $6.77 \mathrm{ppm}$ in 20@13. This corresponds to an upfield shift of $1.55 \mathrm{ppm}$. Similar to signal $\mathbf{a}$, signal $\mathbf{b}$ was also shifted upfield by $1.57 \mathrm{ppm}$. When 20 and $\mathbf{1 3}$ were used in a ratio of 11:1 (Table S1, Entry 4) the signals of $\mathbf{2 0}$ shift to $7.99 \mathrm{ppm}$ and $6.95 \mathrm{ppm}$, while no signals corresponding to the free $\mathbf{2 0}$ are visible. The integrals suggest a $\mathbf{2 0 / 1 3}$ ratio of 3:1. The different ratio is a result of the low solubility of $\mathbf{2 0}$ in $\mathrm{CDCl}_{3}$. At higher 20/13 ratios, signals of encapsulated 20 shift towards the signals of free 20, which indicates that guest-exchange processes occur fast on the time scale of NMR spectroscopy. Furthermore, that might also be an explanation for the fact that 20@13 has not been observed by mass spectrometry so far.

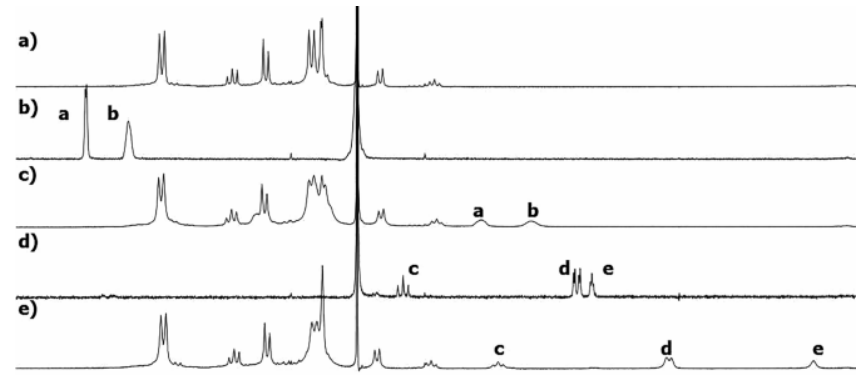

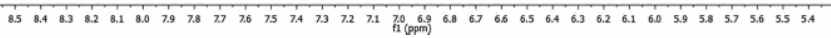

Figure 1. ${ }^{1} \mathrm{H}$ NMR spectra (8.55-5.30 ppm) of encapsulation experiments of 13 with 3-hydroxypyridine (20) and resorcinol (21); a) 13; b) 20 c) 20@13; d) 21; e) 21@13; all spectra recorded in $\mathrm{CDCl}_{3}$ at room temperature.

Inspired by the observation of $\mathbf{2 0 @ 1 3}$ we tested other arenes as possible guests for 13. 2,6-dipyridinecaboxylic acid gave no indication to be encapsulated within 13 (entry 5). The same holds true for pyridine and phenol (entries 6 and 7). We were then wondering if $\mathbf{1 3}$ might be a suitable host for 3-iodopryidine, assuming that a hydrogen bridge between the phenol and 3-iodopryidine may induce a halogen-bonding interaction between the guest and the host. ${ }^{[22]}$ Unfortunately, no indication for such a behaviour or any encapsulation phenomena was observed for 3-iodopryidine (entry 8). Interestingly, also 2hydroxypyridine does not seem to be a suitable guest for $\mathbf{1 3}$ (entry 9). For those compounds, no or only very minor signal shifts ( $\delta=0.03 \mathrm{ppm}$ or less) in the presence of 13 compared to the free compounds were observed (Table 1, entries 5-9). Given these studies, it seems that the guest selection of $\mathbf{1 3}$ is very sensitive with regard to the guest functionalization and its functionalization pattern. This is further supported by the fact that resorcinol (21), which offers a 1,3-difunctionalization similar to 20, is encapsulated by 13 to form $\mathbf{2 1 @ 1 3 ~ ( e n t r y ~ 1 0 ) , ~ w h i l e ~ t h e ~}$ 1,2-difunctionalized catechol gives no clear indication for encapsulation. For comparison, while the signals of $\mathbf{2 0}$ in the presence of 13 shift up to $0.87 \mathrm{ppm}$ compared to the free guest (signals $\mathbf{c}, \mathbf{d}$ and $\mathbf{e}$ in Figure $1 \mathbf{d}$ and e), the signals corresponding to catechol shift by less than $0.10 \mathrm{ppm}$. A full list of all encapsulation experiments and detailed description can be found in the the Supporting Information.

\section{Conclusion}

We have demonstrated a general and modular synthetic approach towards purely organic and hetero-sequenced aza-cyclophanes. To achieve this we used the two key building blocks 2 and 10. The azide-functionalization in those building blocks can be seen as masked amines, suitable to perform a stepwise thermodynamically controlled synthesis of the desired cage compounds. The synthesis of the $2+2+1$ cages $\mathbf{1}$ and $\mathbf{8}$ demonstrates that the precise control of the cage functionalization is possible. The synthesis of $2+2+1$ cage 1 and the $2+1+2$ cage 9 shows that this approach is also suitable to control the substitution pattern. Furthermore, the synthesis of $\mathbf{1 3}$ is an example that our approach is also suitable to synthesis larger cages. In addition, we demonstrated that $\mathbf{1 3}$ can act as a host for functionalized arenes. $\mathbf{1 3}$ is thereby sensitive towards the guests functional groups and the functionalization pattern and prefers thus 1,3-difunctionalized guests.

CCDC 1515608 (for 18) contains the supplementary crystallographic data for this paper. These data can be obtained free of charge from The Cambridge Crystallographic Data Centre.

\section{Acknowledgments}

M. O. thanks the sustainability Scheme of Utrecht University for funding. Johann Jastrezbski, Henk Kleijn and Arnoldus J. van Schaik are acknowledged for technical support. The X-ray diffractometer has been financed by the Netherlands Organization for Scientific Research (NWO).

Keywords: Cage compounds - Combinatorial chemistry Cavity design · Interior functionalization · Host-guest systems

[1] W. Meng, B. Breiner, K. Rissanen, J. D. Thoburn, J. K. Clegg, J. R. Nitschke, Angew. Chem. Int. Ed. 2011, 50, 3479-3483; Angew. Chem. 2011, 123, 3541-3543.

[2] A. Avellaneda, P. Valente, A. Burgun, J. D. Evans, A. W. Markwell-Heys, D. Rankine, D. J. Nielsen, M. R. Hill, C. J. Sumby, C. J. Doonan, Angew. Chem. Int. Ed. 2013, 52, 3746-3749; Angew. Chem. 2013, 125, 3834-3837.

[3] V. Croué, S. Groeb, G. Szalóki, M. Allain, M. Sallé, Angew. Chem. Int. Ed. 2016, 55, 1746-1750; Angew. Chem. 2016, 128, 1778-1782.

[4] P. Mal, B. Breiner, K. Rissanen, J. R. Nitschke, Science 2009, 324, 16971699.

[5] a) M. Yoshizawa, J. K. Klosterman, M. Fujita, Angew. Chem. Int. Ed. 2009, 48, 3418-3438; Angew. Chem. 2009, 121, 3470-3490; b) S. H. A. M. Leenders, R. Gramage-Doria, B. de Bruin, J. N. H. Reek, Chem. Soc. Rev. 2015, 44, 433-448; c) M. Otte, ACS Catal. 2016, 6, 6491-6510.

[6] M. Fujita, D. Oguro, M. Miyazawa, H. Oka, K. Yamaguchi, K. Ogura, Nature 1995, 378, 469-471.

[7] L. J. Barbour, G. W. Orr, J. L. Atwood, Nature 1998, 393, 671-673.

[8] a) D. Samanta, P. S. Mukherjee, Chem. Commun. 2013, 49, 4307-4309; b) D. Preston, J. E. Barnsley, K. C. Gordon, J. D. Crowley, J. Am. Chem. Soc. 2016, 138, 10578-10585; c) W. M. Bloch, Y. Abe, J. J. Holstein, C. M. Wandtke, B. Dittrich, G. H. Clever, J. Am. Chem. Soc. 2016, 138, 1375013755.

[9] G. Zhang, M. Mastalerz, Chem. Soc. Rev. 2014, 43, 1934-1947.

[10] Z. Zhong, B. J. Postnikova, R. E. Hanes, V. M. Lynch, E. V. Anslyn, Chem. Eur. J. 2005, 11, 2385-2394. 
[11] T. J. Mooibroek, M. P. Crump, A. P. Davis, Org. Biomol. Chem. 2016, 14, 1930-1933.

[12] a) S. Otto, R. L. E. Furlan, J. K. M. Sanders, Science 2002, 297, 590-593; b) N. Giuseppone, J.-L. Schmitt, E. Schwartz, J.-M. Lehn, J. Am. Chem. Soc. 2005, 127, 5528-5539; c) B. Içli, N. Christinat, J. Tönnemann, C. Schüttler, R. Scopelliti, K. Severin, J. Am. Chem. Soc. 2009, 131, 3154-3155; d) M. W. Schneider, I. M. Oppel, A. Griffin, M. Mastalerz, Angew. Chem. Int. Ed. 2013, 52, 3611-3615; Angew. Chem. 2013, 125, 3699-3703; e) T. Mitra, K. E. Jelfs, M. Schmidtmann, A. Ahmed, S. Y. Chong, D. J. Adams, A. I. Cooper, Nat. Chem. 2013, 5, 276-281; f) Y. Jin, R. J. Denman, W. Zhang, Chem. Soc. Rev. 2013, 42, 6634-6654; g) K. Acharyya, P. S. Mukherjee, Chem. Eur. J. 2015, 21, 6823-6831.

[13] K. D. Okochi, Y. Jin, W. Zhang, Chem. Commun. 2013, 49, 4418-4420.

[14] B. Chatelet, L. Joucla, J.-P. Dutasta, A. Martinez, V. Dufaud, Chem. Eur. J. 2014, 20, 8571-8574.

[15] a) O. Francesconi, A. Ineco, G. Moneti, C. Nativi, S. Roelens, Angew. Chem. Int. Ed. 2006, 45, 6693-6696; Angew. Chem. 2006, 118, 6845-6848; b) T. J. Mooibroek, J. M. Casas-Solvas, R. L. Harniman, C. M. Renney, T. S. Carter, M. P. Crump, A. P. Davis, Nat. Chem. 2016, 8, 69-74.

[16] a) J. T. A. Jones, D. Holden, T. Mitra, T. Hasell, D. J. Adams, K. E. Jelfs, A. Trewin, D. J. Willock, G. M. Day, J. Bacsa, A. Steiner, A. I. Cooper, Angew.
Chem. Int. Ed. 2011, 50, 749-753; Angew. Chem. 2011, 123, 775-779; b) J. T. A. Jones, T. Hasell, X. Wu, J. Bacsa, K. E. Jelfs, M. Schmidtmann, S. Y. Chong, D. J. Adams, A. Trewin, F. Schiffman, F. Cora, B. Slater, A. Steiner, G. M. Day, A. I. Cooper, Nature 2011, 474, 367-371; c) M. Petryk, J. Szymkowiak, B. Gierczyk, G. Spólnik, Ł. Popenda, A. Janiak, M. Kwit, Org. Biomol. Chem. 2016, 14, 7495-7499.

[17] L. J. Murray, W. W. Weare, J. Shearer, A. D. Mitchell, K. A. Abboud, J. Am. Chem. Soc. 2014, 136, 13502-13505.

[18] D. M. Ermert, I. Ghiviriga, V. J. Catalano, J. Shearer, L. J. Murray, Angew. Chem. Int. Ed. 2015, 54, 7047-7050; Angew. Chem. 2015, 127, 7153-7156.

[19] a) M. Arunachalam, I. Ravikumar, P. Gosh, J. Org. Chem. 2008, 73, 9144 9147 ; b) P. Mateus, R. Delgado, P. Brandão, V. Félix, J. Org. Chem. 2009, 74, 8638-8646.

[20] A. B. Vliegenthart, F. A. L. Welling, M. Roemelt, R. J. M. Klein Gebbink, M. Otte, Org. Lett. 2015, 17, 4172-4175.

[21] O. Francesconi, M. Gentili, C. Nativi, A. Ardá, F. J. Cañada, J. JiménezBarbero, S. Roelens, Chem. Eur. J. 2014, 20, 6081-6091.

[22] L. C. Gilday, T. Lang, A. Caballero, P. J. Costa, V. Félix, P. D. Beer, Angew. Chem. Int. Ed. 2013, 52, 4356-4360; Angew. Chem. 2013, 125, 4452-4456.

Received: January 23, 2017 